\title{
Modelling the optical properties of composite and porous interstellar grains
}

\author{
N. V. Voshchinnikov ${ }^{1,2}$, V. B. Il'in ${ }^{1,2}$, and Th. Henning ${ }^{3}$ \\ 1 Sobolev Astronomical Institute, St. Petersburg University, Universitetskii prosp. 28, St. Petersburg 198504, Russia \\ e-mail: nvv@astro.spbu.ru \\ 2 Isaac Newton Institute of Chile, St. Petersburg Branch, Russia \\ 3 Max-Planck-Institut für Astronomie, Königstuhl 17, 69117 Heidelberg, Germany
}

Received 3 March 2003 / Accepted 16 August 2004

\begin{abstract}
There are indications that interstellar and interplanetary dust grains have an inhomogeneous and fluffy structure. We investigate different methods to describe light scattering by such composite particles. Both a model of layered particles and discrete dipole calculations for particles with Rayleigh and non-Rayleigh inclusions are used.

The calculations demonstrate that porosity is a key parameter for determining light scattering. We find that the optical properties of the layered particles depend on the number and position of layers if the number of layers is small $(\lessgtr 15)$. For a larger number of layers the scattering characteristics become independent of the layer sequence. The optical properties of particles with inclusions depend on the size of inclusions provided the porosity is large. The scattering characteristics of very porous particles with inclusions of different sizes are found to be close to those of multi-layered spheres.

We compare the results of these calculations with the predictions of the effective medium theories (EMT) which are often used in astronomy as a tool to calculate the optical properties of composite particles. The results of our analysis show that the internal structure of grains (layers versus inclusions) only slightly affects the optics of particles provided the porosity does not exceed $50 \%$. It is also demonstrated that in this case the optical properties of composite grains calculated with EMT agree with the results of the exact method for layered particles. For larger porosity, the standard EMT rules (i.e., Garnett and Bruggeman rules) give reliable results for particles with Rayleigh inclusions only.
\end{abstract}

Key words. scattering - dust, extinction - comets - interplanetary medium

\section{Introduction}

Various processes operating in interstellar and circumstellar media are believed to produce inhomogeneous and porous cosmic dust grains (Dorschner 1999; Draine 2003). However, the real structure of interstellar grains remains to be established. The particles may have a layered structure because of the their formation in circumstellar environments (Dominik et al. 1993) and further evolution in molecular clouds (Ehrenfreund 1999; Greenberg 1999). On the other hand, collisions of grains tend to induce coagulation and partial destruction of particles. Because of this, interstellar grains should have inclusions of different size. Therefore, considering the optical properties of cosmic dust grains we are forced to solve the difficult problem of the interaction of radiation with composite particles of different structure.

Fortunately, scientists had felt the necessity to treat the scattering by composite and inhomogeneous particles or media consisting of several components even earlier than the existence of interstellar dust was established. Garnett (1904) was the first to find the averaged (effective) dielectric functions of such a medium assuming that one material was a matrix (host material) in which another material was embedded in the form of small inclusions (so called Maxwell-Garnett mixing rule of the Effective Medium Theory; EMT). Later, Bruggeman (1935) deduced another rule which was symmetric with respect to the materials. These classical mixing rules are still the most popular ones.

Many scientific and applied problems require calculations of light scattering by inhomogeneous particles with good accuracy.

This first became possible at the beginning of the 1950s when the Mie solution for homogeneous spheres was generalized to core-mantle spherical particles in three independent papers (Aden \& Kerker 1951; Shifrin 1952; Güttler 1952). Güttler's solution was used by Wickramasinghe (1963) who first calculated the extinction of layered (graphite core-ice mantle) analogues of cosmic grains.

Mathis \& Whiffen (1989) introduced the first consistent model of composite cosmic grains which were very porous (the volume fraction of vacuum $\sim 80 \%$ ) aggregates of small amorphous carbon, silicate and iron subparticles. The optical properties of such particles were calculated with the Mie theory and EMT. Mathis (1996) updated the composite grain model 
taking into account the abundances of heavy elements obtained for cluster and field B stars and young F, G stars (Snow \& Witt 1996). The new model consisted of three dust grain populations where the visual/near-IR extinction was explained by aggregates with $\sim 45 \%$ vacuum in volume.

Now light scattering computations for inhomogeneous (composite) particles with layers or inclusions from different materials or aggregate particles are often made using the discrete dipole approximation (DDA) or simpler theories (see Voshchinnikov 2004, for discussion). Note that the DDA is a method which still is computationally demanding. Therefore, it is mostly used for illustrative calculations and not for mass production (e.g., Wolff et al. 1994, 1998; Vaidya et al. 2001; Andersen et al. 2003).

The idea to represent composite interstellar grains by multilayered spheres (Voshchinnikov \& Mathis 1999; see also Iatì et al. 2001) has no immediate physical justification ${ }^{1}$ (although such particles may form in stellar envelopes and molecular clouds), but is very attractive as an exact method to calculate the optical properties of composite particles. Such a model permits us to include an arbitrary fraction of any material, and computations do not require large resources. However, the distribution of material inside particles is always spherically symmetric even when its volume fraction tends to zero.

In this paper, we compare the optical properties of composite interstellar grains of various porosity obtained from calculations for layered spheres, pseudospherical particles with inclusions and homogeneous spheres with an effective refractive index. The description of the particle models is given in Sect. 2. We compute different efficiency factors, albedo, etc. and analyze how these quantities depend on the order and number of layers and the size of inclusions (Sect. 3). Special attention is paid to the consideration of very porous grains (Sect. 3.2) because of their particular importance in astronomy, for example, for modelling of comets (Greenberg \& Hage 1991) and the disc of $\beta$ Pictoris (Li \& Greenberg 1998). The possibility to describe the light scattering by porous particles using Mie theory with different EMT rules is studied in Sect. 4. Concluding remarks are presented in Sect. 5 .

\section{Models of composite grains}

Processes operating in the winds of late-type stars such as grain nucleation and growth, shattering in the diffuse interstellar medium, and finally coagulation and accretion in molecular clouds and protoplanetary disks lead certainly to dust grains with rather irregular shapes and very complicated internal structure (Dorschner \& Henning 1995). The details of the grain interiors are not directly important for surface chemistry, but the optical behaviour of the particles may be a strong function of this structure. Direct evidence for the structure of

\footnotetext{
${ }^{1}$ B. Michel (1995, J. Opt. Soc. Am. A, 12, 2471) has shown that the spherical multi-layered particles are the lowest order approximation to the strong-permittivity fluctuations theory, i.e., an ensemble of randomly oriented, arbitrarily oriented particles is equivalent to a multi-layered particle, if spatial correlations inside the particles can be neglected.
}
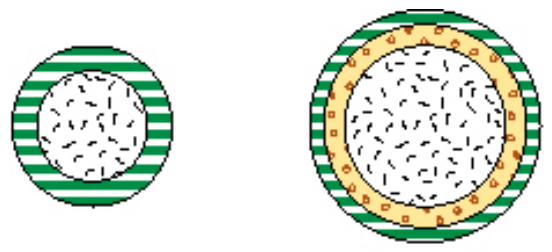

core-mantle

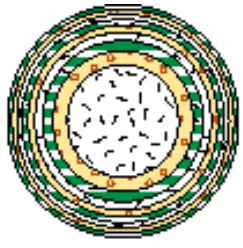

9-layered

Fig. 1. The cut of the spherical particles by the plane. The models of composite grains containing the same amount of carbon and silicate are shown. The 3- and 9-layered spheres consist of equivolume spherical layers with the total volume fractions of carbon, silicate and vacuum equal to $33.33 \%$. The core-mantle particle includes the same mass of carbon and silicate but is free of vacuum.
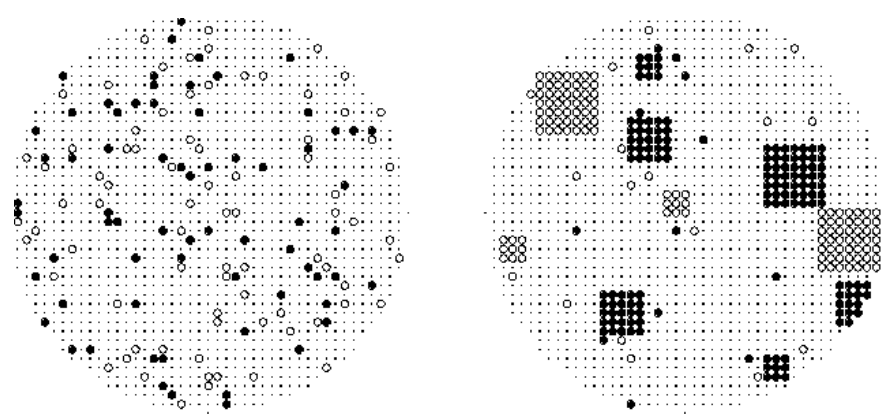

Fig. 2. The cut of the pseudospherical particles with the maximum size $d_{\max }=45$ by the plane. The models of very porous particles with small single size (left) and different size (right) cubic inclusions are shown. The volume fractions of carbon and silicate are equal to 5\%.

these particles is difficult to obtain with the exception of interplanetary dust grains collected in the upper atmosphere and the solar system. Therefore, a more general attempt to describe the particles and to explore changes in their optical properties is required.

A frequently used approach in astronomy is the modelling of inhomogeneous grains by two-layered (core-mantle) spheres and particles with voids or inclusions using EMT-Mie calculations. In this paper, we consider layered particles and particles with inclusions as the models for the description of the optical properties of the inhomogeneous or composite grains. The amount of a material in such particles is determined by its volume fraction $V_{i}\left(\Sigma_{i} V_{i} / V_{\text {total }}=1\right)$. The particle porosity $\mathcal{P}(0 \leq \mathcal{P}<1)$ is introduced as

$\mathcal{P}=V_{\text {vac }} / V_{\text {total }}=1-V_{\text {solid }} / V_{\text {total }}$,

where $V_{\text {vac }}$ and $V_{\text {solid }}$ is the volume fraction of vacuum and solid material, respectively.

In our calculations presented below, composite particles of several materials are considered. The refractive indices for them were taken from the Jena-Petersburg Database of Optical Constants (JPDOC) which was described by Henning et al. (1999) and Jäger et al. (2003).

Carbon and silicates are the materials most often used in cosmic dust models (see Mathis et al. 1977; Draine \& Lee 1984, and so on). We consider the particles composed of amorphous carbon (AC1), astronomical silicate (astrosil) and vacuum with varied volume fraction of each constituent. 
The optical constants for AC1 $(m=1.98+0.23 i)$ and astrosil $(m=1.68+0.03 i)$, corresponding to the wavelength $\lambda=0.55 \mu \mathrm{m}$, were taken from the papers of Rouleau \& Martin (1991) and Laor \& Draine (1993), respectively.

\subsection{Layered particles}

Here we represent composite grains by particles consisting of many concentric spherical homogeneous layers of cyclically changing materials. Such a model is not primarily meant as a physical description of the actual grain structure, but as a possibility for describing light scattering by inhomogeneous particles of complex structure.

Vacuum can be one of the materials, and a composite particle may have a central cavity or voids in the form of concentric layers. This model allows one to include at any position inside a spherical particle any fraction of a material (from extremely small to very large). The light scattering calculations are based on the exact theory which is true for particles of any size and refractive index.

The schematic representation (cross cut) of layered spherical grains is given in Fig. 1. The particles are composed of a specified number of concentric spherical homogeneous layers. The order of the layers and their total number can be specified separately. Following Voshchinnikov \& Mathis (1999), we assume further that different material layers cyclically change inside a particle and call the repeating set of such layers a shell. This means that a particle consists of a specified number of concentric shells, and the simplest model particle contains one shell of two materials. The core-mantle particle presented in Fig. 1 does not contain vacuum, but its mass is the same as that of the other two particles shown. As a result, its volume is less by factor of $1 / 3$ and the outer radius by $\sqrt[3]{1 / 3} \approx 0.69$, respectively.

The formal solution to the light scattering problem for $n$-layered spheres can be easily written in matrix form with the separation of variables method (see, e.g., Kerker 1969). In this case the scattering coefficients are calculated as the ratios of two determinants of order $2 n+1$ containing Riccati-Bessel functions and their first derivatives of real and complex arguments. However, for practical reasons, it is better to use the recursive algorithm developed by Wu \& Wang (1991) and Johnson (1996). In order to make calculations for highly absorbing particles of large sizes, one should take into account the modifications suggested by Wu et al. (1997) and Gurwich et al. (2001).

\subsection{Particles with inclusions}

The optical properties of particles with inclusions can be estimated on the basis of rather complicated calculations (see, e.g., Wolff et al. 1994; Videen \& Chýlek 1998) or from laboratory measurements (Kolokolova \& Gustafson 2001). If the volume fraction of inclusions is not very large ( $\$ 10 \%)$, the EMT-Mie calculations give the results with good accuracy (Wolff et al. 1994, 1998; Kolokolova \& Gustafson 2001).

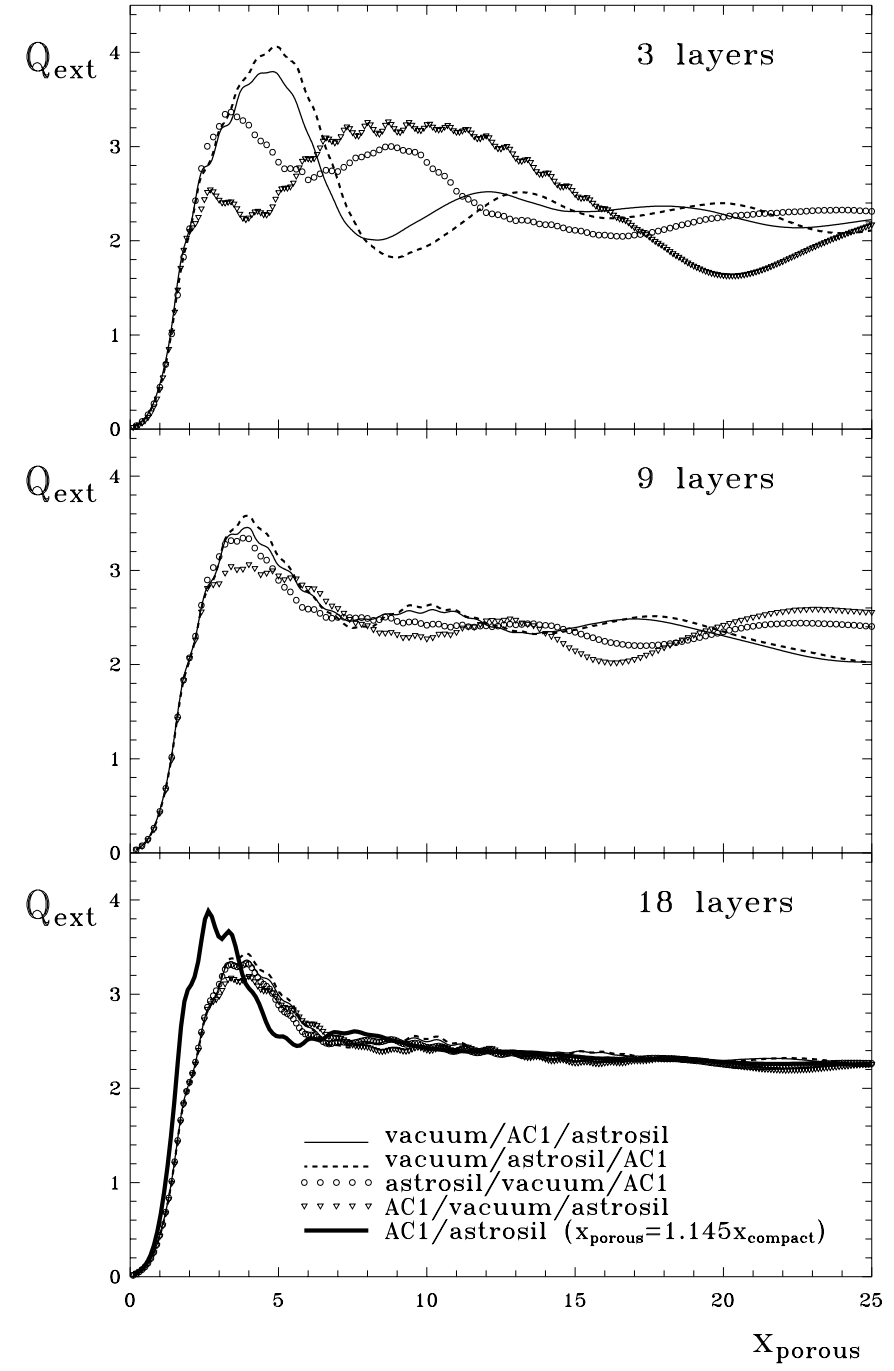

Fig. 3. Size dependence of the extinction efficiency factors for layered spherical particles. Each particle contains an equal fraction of amorphous carbon (AC1), astrosil and vacuum (the porosity $\mathcal{P}=1 / 3$ ) separated in equivolume layers. The cyclic order of the different material layers is indicated (starting from the core). The effect of the increase of the number of layers is illustrated. The thick line at the lowest panel corresponds to compact spheres consisting of $\mathrm{AC} 1$ and astrosil. For a given value of the size parameter, the compact and porous particles have the same mass.

However, our goal is the consideration of particles with an arbitrary amount of inclusions and different porosity. Therefore, the calculations are performed with the discrete dipole approximation (DDA). We use the last version of the DDA program (DDSCAT 6.0) developed by Draine \& Flatau (2003). This technique can treat particles of arbitrary shapes and/or of inhomogeneous structure. A detailed review of the DDA and its applications is given by Draine (2000).

The particles ("targets" in the DDSCAT terminology) are constructed using two special routines. One routine produces spherical targets with inclusions of a fixed size, while another creates targets with a given distribution of inclusions over their sizes. Both routines produce first a cube with randomly distributed cubic inclusions. The sizes of the target $d_{\max }$ and of the inclusions $d_{\text {incl }}$ are expressed in units of the interdipole 

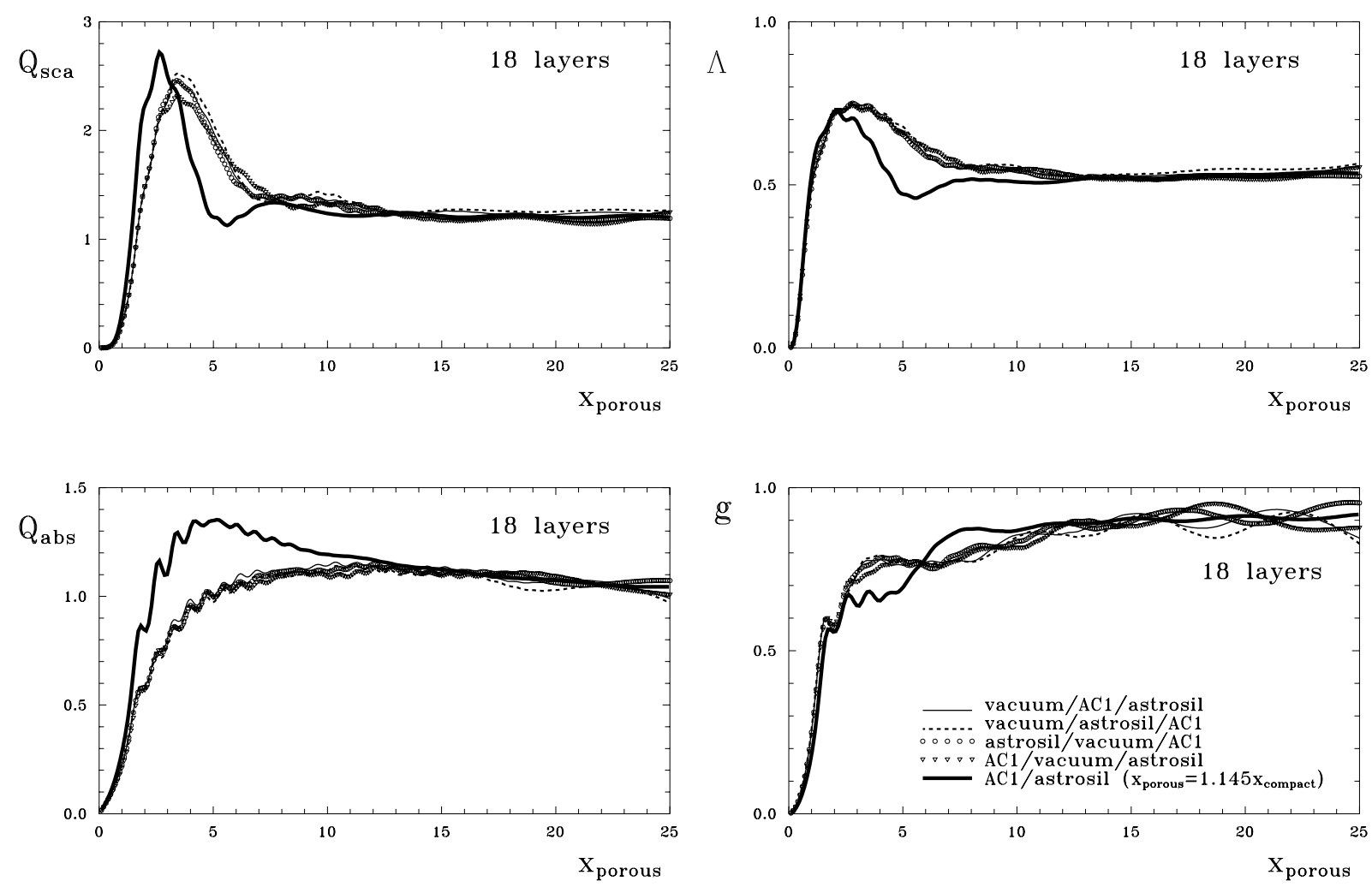

Fig. 4. Size dependence of the scattering and absorption efficiency factors, albedo and asymmetry parameter for multi-layered spheres. The parameters of particles are the same as in Fig. 3.

distance $d$. In the cube, we just inscribe a sphere and remove all inclusions and their parts being outside the sphere.

In contrast to previous modelling (Henning \& Stognienko 1993; Lumme \& Rahola 1994; Wolff et al. 1994, 1998; Vaidya et al. 2001), porous particles are not produced by removing dipoles or inclusions from a target but by attributing the refractive index $m=1.000001+0.0 i$ to them. We believe that such a structure better corresponds to cosmic aggregates.

For the purpose of treating very porous particles, the number of dipoles in pseudospheres is taken quite large. In all cases considered, the particles with the maximum size $d_{\max }=91$ are studied. This value corresponds to the total number of dipoles in pseudospheres $N_{\text {dip }}=357128-381915$ depending on the size of inclusions $d_{\text {incl }}$. Thus, the criterion of the validity of the DDA for extinction/scattering cross sections $|m| k d<1^{2}$ of Draine \& Flatau (2003) $(k=2 \pi / \lambda$ is the wavenumber with $\lambda$ being the wavelength in vacuum) is satisfied up to the size parameter $x_{\text {porous }}=2 \pi r / \lambda \approx 27-28$.

Targets with the values of $d_{\text {incl }}$ ranging from 1 to 9 are considered. The resulting structure (cross cut) of pseudospherical grains is shown in Fig. 2. Note that the inclusions of the size $d_{\text {incl }}=1$ are dipoles, while the inclusions with $d_{\text {incl }}=3,5,7$ and 9 consist of 27, 125, 343 and 729 dipoles, respectively.

The optical characteristics of pseudospheres with inclusions were averaged over three targets obtained for different random number sets. The calculations show that in our case

\footnotetext{
2 Note that sometimes the fulfillment of this criterion does not guarantee the correct result (see, e.g., discussion in Andersen et al. 2003).
}

such an approach is practically equivalent to time-consuming numerical averaging over target orientations.

\section{Towards unified optical properties}

It is evident that composite particles of various structure and shape should exist in space. Here we focus our attention on spheres of different porosity because of their frequent use in the modelling of interstellar and cometary grains and possible importance in attacking the problem of cosmic abundances. Our analysis should also help to estimate the range of validity of some previous models and to clarify the physical background of them.

\subsection{Particles of moderate porosity}

We start with the consideration of layered particles. Figure 3 shows the extinction efficiency factors $Q_{\mathrm{ext}}=C_{\mathrm{ext}} / \pi r_{\mathrm{s}}^{2}\left(C_{\mathrm{ext}}\right.$ is the extinction cross section, $r_{\mathrm{s}}$ the outer radius) of layered spheres. The optical properties of core-mantle spheres have been studied rather well and seem to show no significant peculiarities $^{3}$ (Babenko et al. 2003). In contrast, three-layered spheres already can produce anomalous extinction of light. The order of the materials strongly affects the behaviour of extinction for such particles (the upper panel of Fig. 3). First of all, the location of vacuum (the core or the middle layer) is important. The curve for particles with a carbon core and an

\footnotetext{
3 Except for a resonance peak arising for particles with mantles having a large refractive index (see, e.g., Gurwich et al. 2003).
} 
$Q_{\text {ext }}$

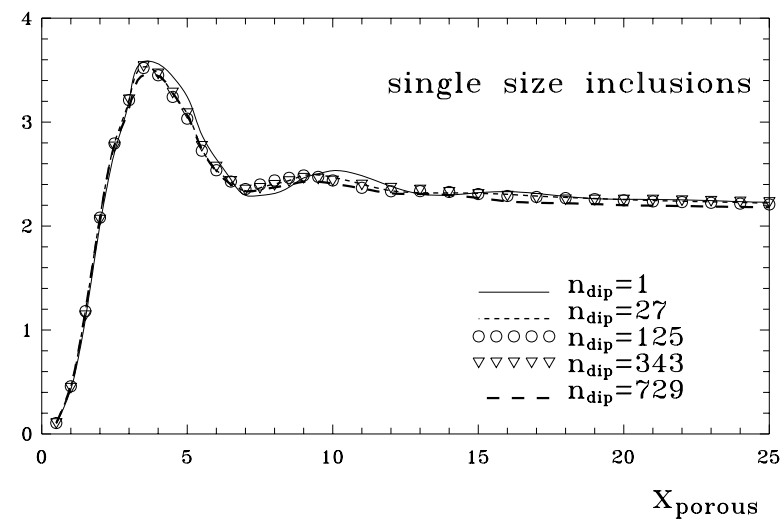

Fig. 5. Size dependence of the extinction efficiency factors for pseudospheres with inclusions of the same single size after averaging of three different targets. Each particle contains an equal volume fraction $(33.33 \%)$ of $\mathrm{AC} 1$, astrosil and vacuum. The effect of variations of the size of inclusions is illustrated.

outermost astrosil layer is the most peculiar curve. Here, a very rare situation is observed: the first maximum is damped, but there is a very broad second maximum. Note that the scattering efficiency depends more strong on the order of layers than the absorption efficiency. However, all the peculiarities disappear when the number of layers increases: the difference between the curves becomes rather small for particles with 9 layers ( 3 shells) and is hardly present for particles with 18 layers (6 shells; see Figs. 3 and 4). Figure 4 shows the size dependence of the scattering $\left(Q_{\text {sca }}\right)$ and absorption $\left(Q_{\text {abs }}\right)$ efficiency factors, albedo $\Lambda=Q_{\text {sca }} / Q_{\text {ext }}$ and the parameter $g^{(4)}$ describing the asymmetry of the phase function $F(\Theta, \Phi)$

$g=\langle\cos \Theta\rangle=\frac{\int_{4 \pi} F(\Theta, \Phi) \cos \Theta \mathrm{d} \omega}{\int_{4 \pi} F(\Theta, \Phi) \mathrm{d} \omega}$

for multi-layered spheres. As has been noted by Voshchinnikov \& Mathis (1999): the optical properties weakly depend on the order of materials in each of the shells and are close to some "average" properties, if a particle consists of many shells ( $\gtrsim 3-5)$. In other words, for such particles, different efficiency factors as well as albedo $\Lambda$ and the asymmetry parameter $g$ depend practically only on the volume fraction of materials.

The solid thick lines in Figs. 3 and 4 show the size dependence of the optical characteristics of compact $(\mathcal{P}=0)$ spheres consisting of the same amount of solid materials as the porous ones. To compare the optical properties of porous and compact particles, it is helpful to normalize the size parameter of either the compact or porous particle using the relation

$x_{\text {porous }}=\frac{x_{\text {compact }}}{(1-\mathcal{P})^{1 / 3}}=\frac{x_{\text {compact }}}{\left(V_{\text {solid }} / V_{\text {total }}\right)^{1 / 3}}$.

In the case of the particles presented in Figs. 3 and 4, this leads to stretching of the $x$ scale for compact particles by a factor of $\sqrt[3]{3 / 2} \approx 1.145$. It can be seen that the presence of vacuum inside the composite particles reduces the peak of the absorption

${ }^{4}$ The notation $g$ is related to the Henyey-Greenstein phase function that is very often use in radiative transfer modelling.

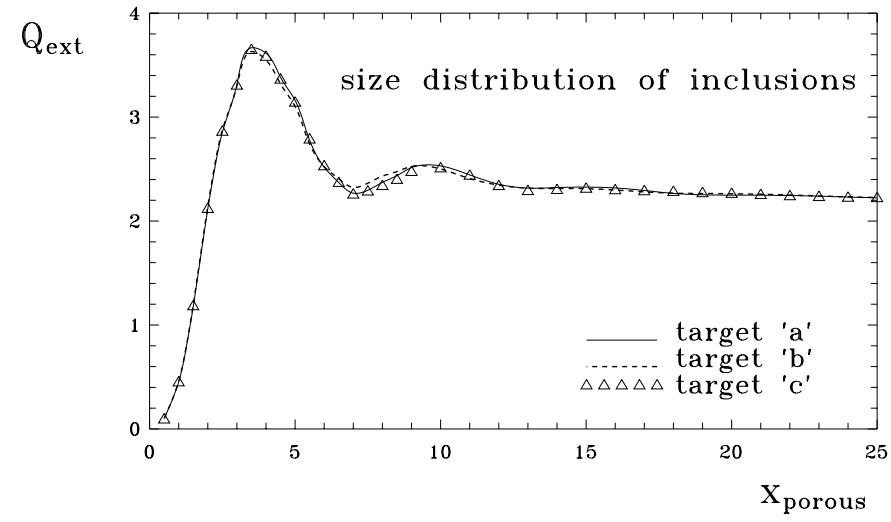

Fig. 6. Size dependence of the extinction efficiency factors for pseudospheres with a size distribution of inclusions. Each particle contains an equal volume fraction $(33.33 \%)$ of $\mathrm{AC} 1$, astrosil and vacuum. The volume fractions of inclusions of different sizes are approximately the same.

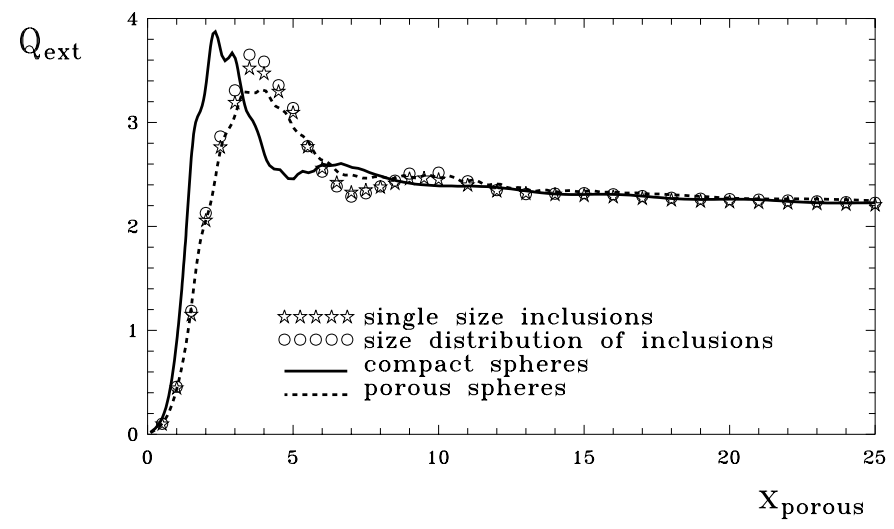

Fig. 7. The average extinction efficiencies for particles with single size inclusions (from Fig. 5) and particles with size distribution of inclusions (from Fig. 6). Each particle contains an equal volume fraction $(33.33 \%)$ of $\mathrm{AC} 1$, astrosil and vacuum. The thick solid line corresponds to compact spheres consisting of $\mathrm{AC} 1$ and astrosil. For a given value of the size parameter, the compact and porous particles have the same mass. The thick dashed line shows the extinction for layered spheres after averaging over four samples presented at the bottom panel of Fig. 3.

efficiency (Fig. 4, left lower panel) and shifts that of the scattering efficiency (Fig. 4, left upper panel). Correspondingly, these two effects explain the behaviour of the curves for the extinction (Fig. 3). A medium porosity influences the albedo and the asymmetry parameter only in a restricted range of size parameters.

Similar calculations for particles with inclusions were performed many times using the DDA technique. But so far only Wolff et al. $(1994,1998)$ considered particles with large (non-Rayleigh) inclusions. They computed the optical properties of silicate spheres and spheroids with a size parameter up to $x_{\text {porous }}=10$ and a volume fraction of vacuum inclusions up to $80 \%$. For the efficiency factors and asymmetry parameter, the difference between particles with Rayleigh ${ }^{5}$ and

\footnotetext{
${ }^{5}$ In this case, the size of the inclusion is much smaller than the radiation wavelength in the medium.
} 

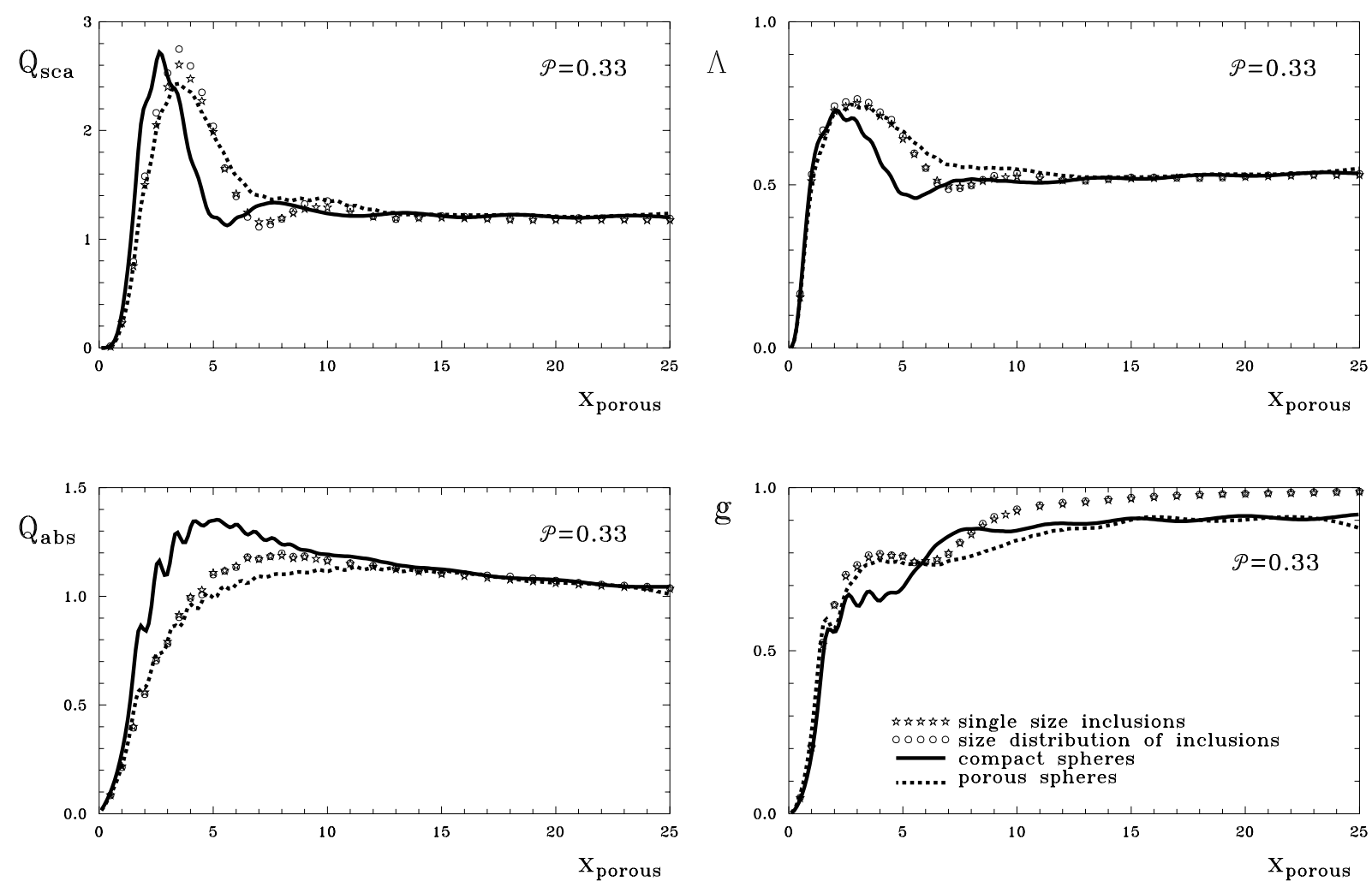

Fig. 8. Size dependence of the scattering and absorption efficiency factors, albedo and the asymmetry parameter for pseudospheres with inclusions. The parameters of particles are the same as in Fig. 7.

non-Rayleigh vacuum inclusions became noticeable for the porosity $\mathcal{P} \gtrsim 0.4$.

The results of our DDA calculations of the extinction efficiency factors for pseudospheres with the porosity $\mathcal{P}=0.33$ are shown in Figs. 5-7. The volume fractions of the materials are approximately the same as in the particles presented in Figs. 3 and 4 but the materials are present in the form of inclusions instead of layers (vacuum is considered as a matrix). Figure 5 shows the results obtained for particles with single size inclusions. Note that despite the different structure of the targets (the number of inclusions reduces from $\sim 240000$ to $\sim 330$ with growing $n_{\text {dip }}$ ), the differences between the extinction efficiencies are quite small. The same conclusion is correct for particles having inclusions of different sizes. The results for three different targets with a distribution of inclusions of five sizes are shown in Fig. 6. The size of the inclusions ranges from 1 to 9 and the number of inclusions is inversely proportional to their volume. In other words, the total numbers of dipoles in the inclusions of each size are approximately the same.

The extinction efficiencies of the particles with inclusions are compared with those of layered particles in Fig. 7. The difference between compact and porous particles is clearly seen, but the results for porous particles with inclusions and layers look rather similar (excluding, perhaps, the height of the first maximum). This behaviour is confirmed by Fig. 8 where other efficiencies, albedo and the asymmetry parameter are presented. The largest deviations occur for the scattering efficiency $Q_{\text {sca }}$ (in the range $x_{\text {porous }} \approx 3-10$ ) and the asymmetry parameter $g$ for the size parameter $x_{\text {porous }} \gtrsim 10$. The latter does not seem to be an artifact related to small number of angles used in our calculations during the averaging over scattering.

Thus, we can conclude that if materials are "well mixed" inside a particle of intermediate porosity, its internal structure in form of layers, Rayleigh or non-Rayleigh inclusions hardly can be inferred from the transmitted radiation. In contrast, there is a clear difference between the optical properties of compact and porous grains.

\subsection{Very porous particles}

It is commonly accepted that the fraction of vacuum in interstellar dust grains can be large. For example, very porous particles are often used to model cometary grains and dust in protoplanetary discs. Greenberg \& Hage (1991) claim that the porosity of dust aggregates in comets can be in the range $0.93<\mathcal{P}<0.98$. Their conclusion is based on the model of porous grains developed by Hage \& Greenberg (1990) who used a volume integral equation method similar to the DDA for light scattering calculations. A verification of this method had been made only for small compact spheres, but the method was applied to large and very porous particles. A qualitative agreement between the results obtained with this method and from Garnett-Mie calculations was found. In both cases the absorption cross section $C_{\text {abs }}$ increased and albedo $\Lambda$ decreased when the porosity grew. Although the validity of these conclusions for particles beyond the Rayleigh domain remains unclear, the results of Hage \& Greenberg (1990) are frequently 


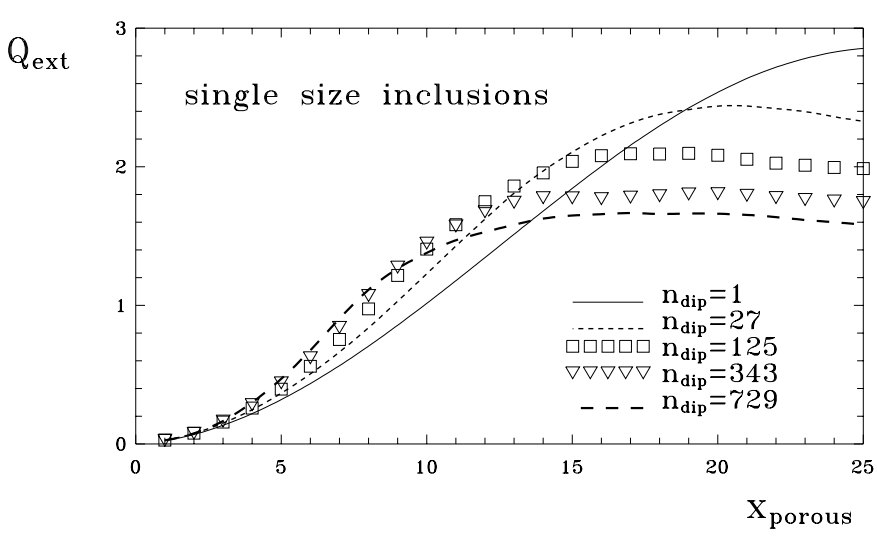

Fig. 9. Size dependence of the extinction efficiency factors for very porous pseudospheres with inclusions of different size. Each particle contains volume fractions of $\mathrm{AC} 1$ and astrosil equal to about 5\%, the porosity $\mathcal{P}=0.9$. The particles are similar to those presented in Fig. 5 but have larger porosity.

used for estimates of grain properties in comets (see, e.g., Mason et al. 2001).

Here we analyze in detail highly porous particles in the case of $\mathcal{P}=0.9$. The results are presented in Figs. 9-11 in a manner similar to that used in Figs. 5-7. We should note the problem connected with the construction of targets when the size of inclusions was large. In this case, the total number of dipoles from AC1 and astrosil was 36000-39000 and they were located in $\sim 50$ inclusions which might not always touch others. Nevertheless, the changes of the general behaviour of extinction with an increase of the number of dipoles in inclusions are clearly seen in Fig. 9. The extinction efficiency factors $Q_{\text {ext }}$ become larger for small values of $x$ and smaller for large ones. This essentially deviates from what was observed for particles of intermediate porosity (cf. Fig. 5). The growth of porosity leads to the disappearance of the first maximum. But the curves for particles with inclusions of large sizes do not approach the limiting value $Q_{\text {ext }}=2$ defined by the "extinction paradox". This fact should be related to special topology of very porous particles with large inclusions (values of $n_{\text {dip }}$ ). Possibly, some inclusions intercept a part of light scattered from other inclusions and scatter it in the forward direction. This decreases the extinction.

However, if particles are composed of subparticles of different size, their extinction is similar to the usual one and the factors $Q_{\text {ext }} \rightarrow 2$ if $x \rightarrow \infty$ (see Fig. 10). The same conclusion is valid for extinction produced by an ensemble of particles with inclusions of single sizes presented in Fig. 11. This figure together with Fig. 12 demonstrates a very important result: the optical properties of very porous layered particles and particles with inclusions are similar. Note that in both cases the models of particles were constructed in such a manner that the materials inside them were "well mixed", i.e. the location of inclusions in the form of layers or islands is not distinguished.

This leads to the interesting conclusion that a very simple computational model of multi-layered particles seems to be of possible use in treating the optics of composite grains.

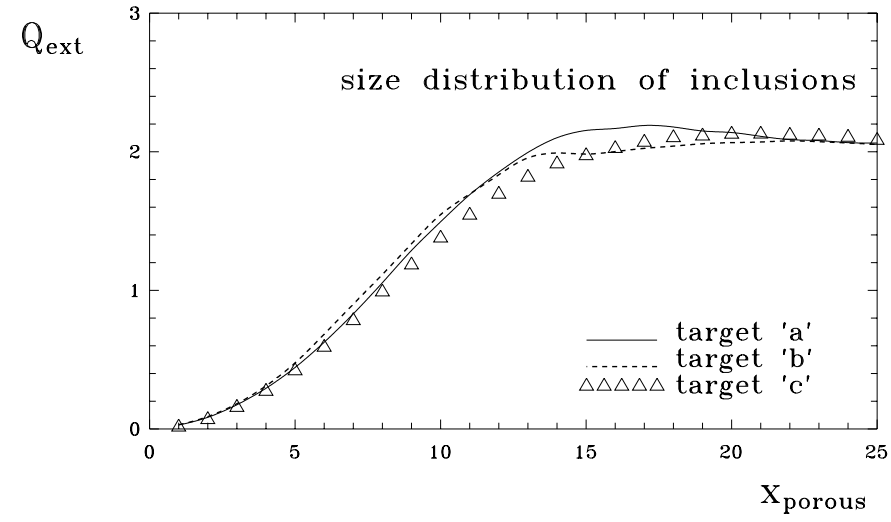

Fig. 10. Size dependence of the extinction efficiency factors for very porous pseudospheres with a size distribution of inclusions. Each particle contains volume fractions of $\mathrm{AC} 1$ and astrosil equal to about $5 \%$, the porosity $\mathcal{P}=0.9$. The particles are similar to those presented in Fig. 6 but have larger porosity.

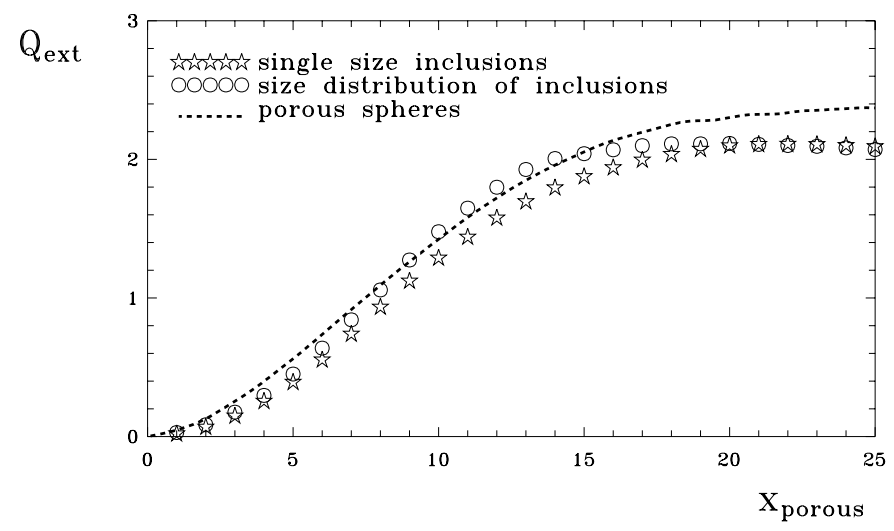

Fig. 11. Averaged extinction efficiencies for particles with single size inclusions (from Fig. 9) and particles with a size distribution of inclusions (from Fig. 10). Each particle contains volume fractions of AC1 and astrosil equal to about $5 \%$, the porosity $\mathcal{P}=0.9$. The particles are similar to those presented in Fig. 7 but have larger porosity. The thick dashed line shows the extinction for layered spheres.

\subsection{Particles of different porosity}

The role of porosity in dust optics can be properly analyzed using the normalized cross sections

$$
\begin{aligned}
C^{(\mathrm{n})} & =\frac{C(\text { porous grain })}{C(\text { compact grain of same mass })} \\
& =(1-\mathcal{P})^{-2 / 3} \frac{Q \text { (porous grain })}{Q \text { (compact grain of same mass })} .
\end{aligned}
$$

The quantity $C^{(\mathrm{n})}$ shows how porosity increases or decreases the cross section. Such an investigation has been performed by Krügel \& Siebenmorgen (1994) for absorption cross sections of small particles with $x<1$. They calculated the effective optical constants of porous particles using, in particular, the Bruggeman mixing rule, and applied the Mie theory to get $Q_{\text {abs. }}$. Krügel \& Siebenmorgen find that the cross sections $C_{\text {abs }}^{(\mathrm{n})}$ increase with $\mathcal{P}$ until $\mathcal{P} \lesssim 0.6$ and then decrease.

Figure 13 shows the normalized extinction cross sections computed for spheres of different porosity. The results are plotted in the scale related to the size parameter $x_{\text {compact }}$ calculated 

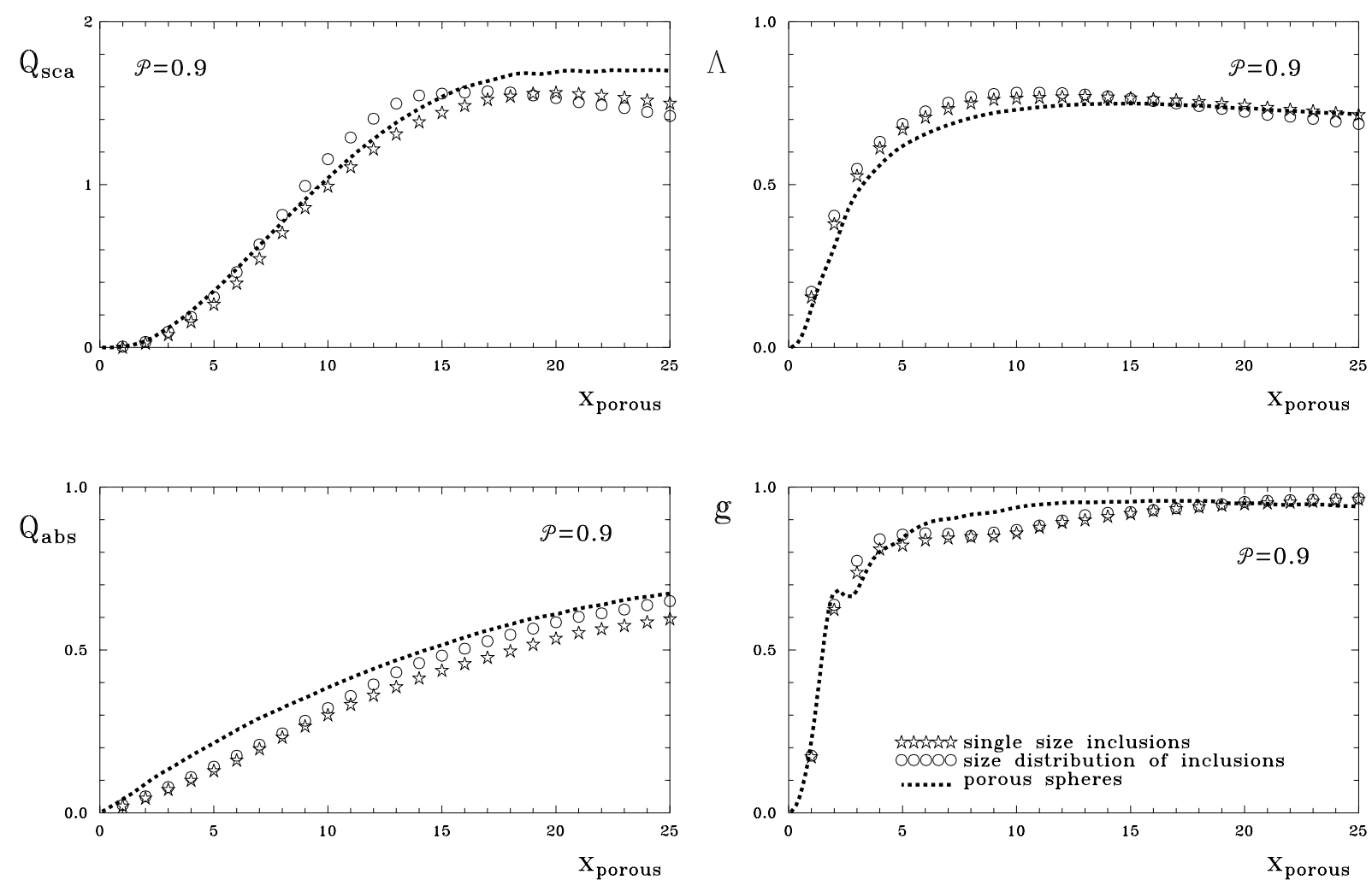

Fig. 12. Size dependence of the scattering and absorption efficiency factors, albedo and the asymmetry parameter for pseudospheres with inclusions. The parameters of particles are the same as in Fig. 11.

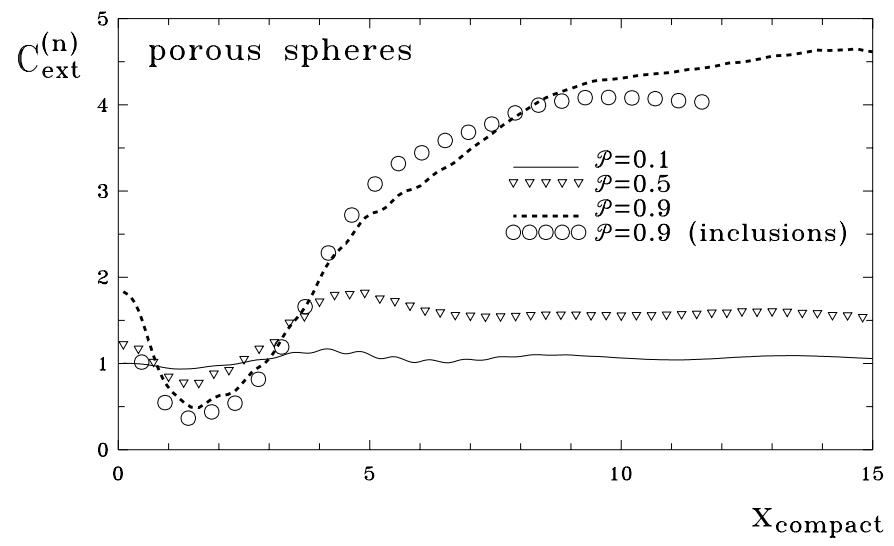

Fig. 13. The normalized extinction cross sections for multi-layered spheres of different porosity. Open circles show the normalized extinction for pseudospheres with a size distribution of inclusions (see Fig. 10).

according to Eq. (3). Since the extinction factors generally decrease when $\mathcal{P}$ increases (cf. the lower panel of Figs. 3 and 11), the values of $C_{\mathrm{ext}}^{(\mathrm{n})}$ are greater than unity if the size parameter is smaller than $\sim 1$ or larger than $\sim 3$. Thus, the porosity increases the extinction of small and large ${ }^{6}$ particles. An opposite case is observed only in a restricted range of the size parameters

\footnotetext{
${ }^{6}$ For very large particles, the normalized cross sections approach to asymptotic values $C^{(\mathrm{n})} \rightarrow(1-\mathcal{P})^{-2 / 3}$ (see Eq. (4)) which are equal to 4.64 and 1.59 if $\mathcal{P}=0.9$ and 0.5 , respectively.
}

$x_{\text {compact }} \approx 1-3$ where the extinction by compact spheres has a maximum (see, e.g., Fig. 7).

As follows from Fig. 14, such a behaviour of the normalized extinction cross sections is accompanied by similar changes of the scattering and absorption cross sections. At the same time, the scattering and absorption efficiencies sharply and slightly grow with $x$ for very porous grains of large sizes. Note also that both $\Lambda$ (beginning with $x_{\text {compact }} \gtrsim 2-3$ ) and $g$ (for particles of all sizes) increase with porosity. The behaviour of $C_{\mathrm{abs}}^{(\mathrm{n})}$ and $\Lambda$ found by us is more complicated than predicted by Hage \& Greenberg (1990). Namely, the growth of porosity leads to an increase of $C^{(n)}$ and a decrease of $\Lambda$ for very small size parameters, and to an increase of both quantities for large values of $x$. There exists also a small interval of intermediate size parameters where both $C^{(\mathrm{n})}$ and $\Lambda$ decrease.

Therefore, we can expect larger extinction, scattering and absorption of radiation by porous particles with radius $r_{\mathrm{s} \text {,compact }}$ at wavelengths $\lambda \lesssim 2 / 3 \pi r_{\mathrm{s} \text {,compact }}$ and $\lambda \gtrsim 2 \pi r_{\mathrm{s} \text {,compact }}$ in comparison with compact particles of the same mass. At the intermediate wavelengths, the compact particles absorb and scatter more radiation. For example, the "importance" of compact grains in the production of extinction is larger at the near$\mathrm{UV} /$ visual range of wavelengths $(0.21 \mu \mathrm{m} \lesssim \lambda \lesssim 0.63 \mu \mathrm{m})$ and at the near-IR wavelengths $(2.1 \mu \mathrm{m} \lesssim \lambda \lesssim 6.3 \mu \mathrm{m})$ if $r_{\mathrm{s}, \text { compact }}=0.1 \mu \mathrm{m}$ and $r_{\mathrm{s}, \text { compact }}=1 \mu \mathrm{m}$, respectively. Note that the latter estimates are rather approximate because of the wavelength dependence of the refractive index of materials (see Voshchinnikov et al. 2004, for more details). 

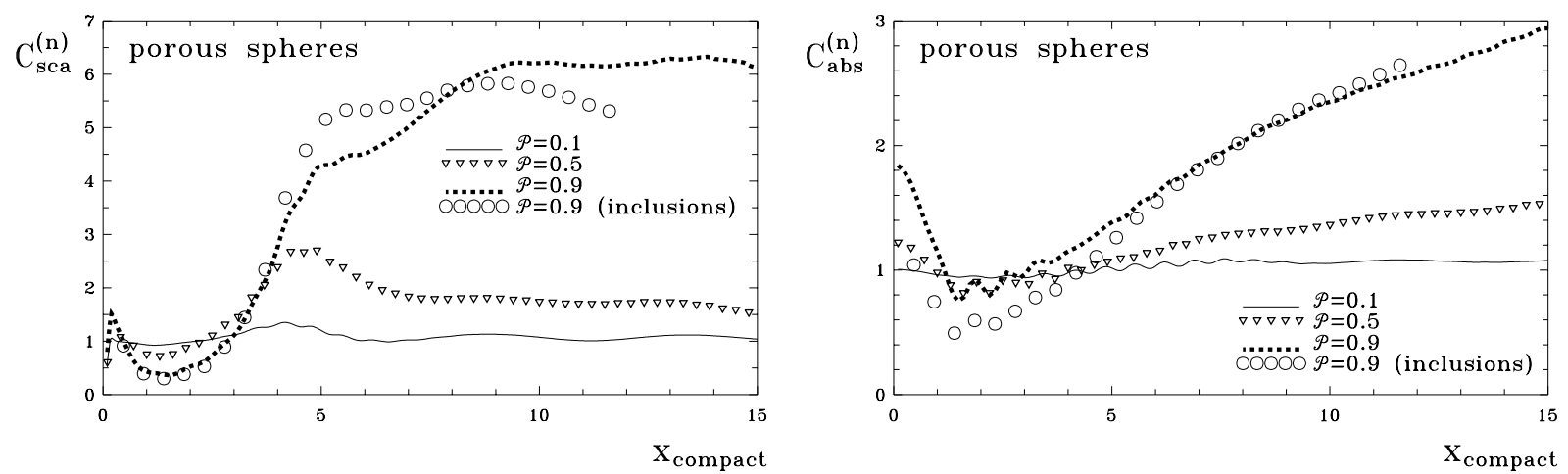

Fig. 14. Size dependence of the scattering and absorption normalized cross sections for multi-layered porous spheres and pseudospheres with a size distribution of inclusions. The parameters of particles are the same as in Fig. 13.
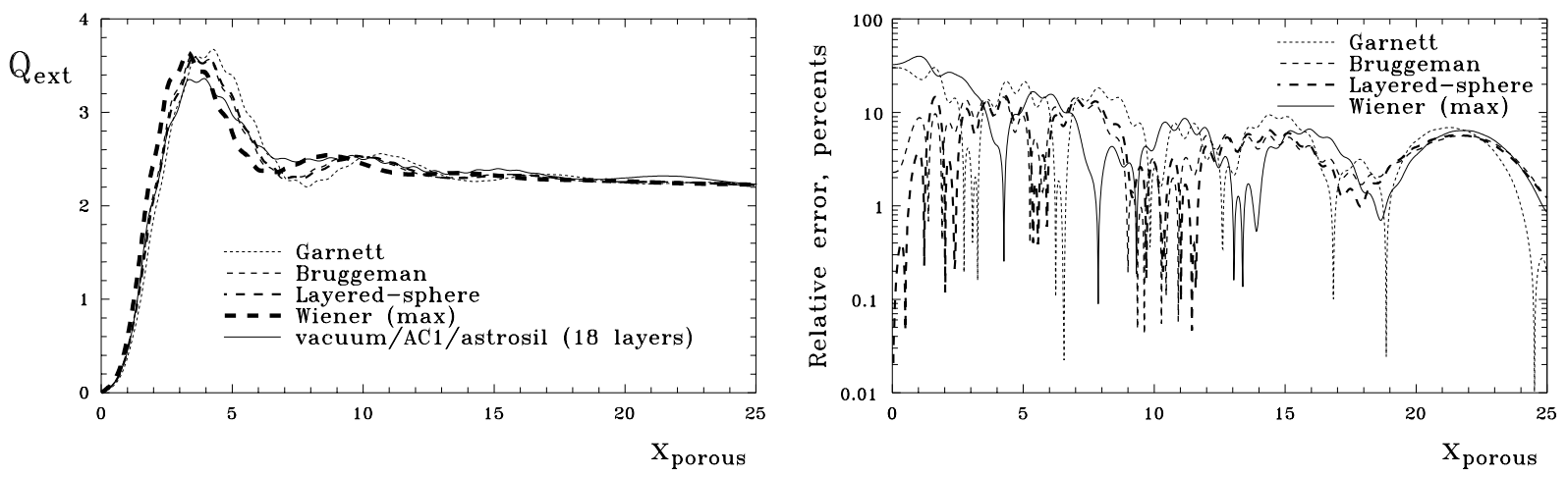

Fig. 15. Size dependence of the efficiency factors (left panel) and their relative errors (right panel) calculated with the exact theory for multilayered spheres and with the Mie theory using four different EMT rules. Multi-layered particles contain an equal volume fraction of amorphous carbon (AC1), astrosil and vacuum. The cyclic order of the 18 layers is indicated.

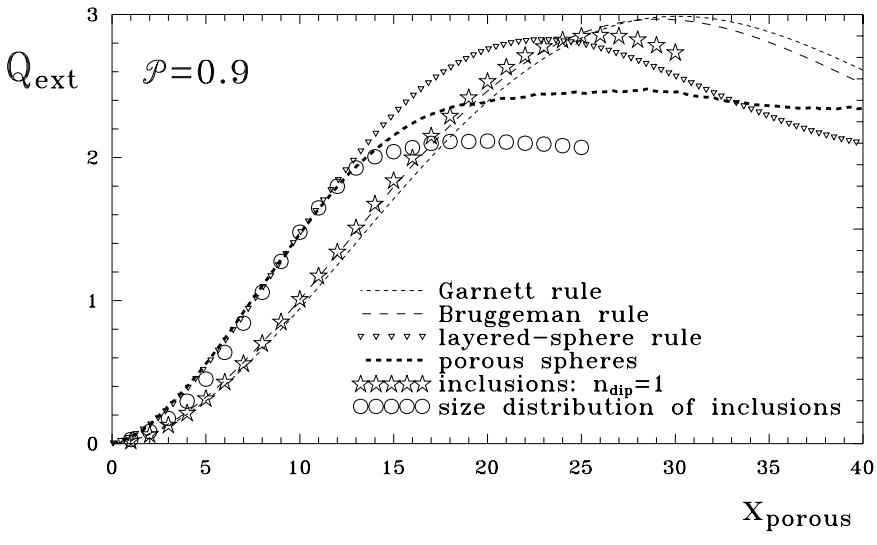

Fig. 16. Size dependence of the extinction efficiency factors calculated for multi-layered spheres, pseudospheres with inclusions and with the Mie theory using three different EMT rules. The porosity of particles is $\mathcal{P}=0.9$.

\section{Comparison with Effective Medium Theory}

The EMT is an approach to treat inhomogeneous scatterers as homogeneous particles having an average (effective) refractive index. The attempts to find the "best" EMT mixing rule continue up to now (e.g., Maron \& Maron 2004). The EMT is well described in the recent reviews of Sihvola (1999), Chýlek et al. (2000) and papers of Spanier \& Herman (2000) and Kolokolova \& Gustafson (2001). There are many EMT rules, but besides a few ones they are rather similar in principle.
Here we give formulas of the most often used EMT rules for $n$-component mixtures: the Garnett (1904) and Bruggeman (1935) rules. In the first case, the mixing rule averages the dielectric permittivities of inclusion materials $\varepsilon_{i}$ and a "matrix" (host) material $\varepsilon_{\mathrm{m}}{ }^{7}$

$\varepsilon_{\mathrm{eff}}=\varepsilon_{\mathrm{m}}\left(1+\frac{3 \sum_{i} f_{i} \frac{\varepsilon_{i}-\varepsilon_{\mathrm{m}}}{\varepsilon_{i}+2 \varepsilon_{\mathrm{m}}}}{1-\sum_{i} f_{i} \frac{\varepsilon_{i}-\varepsilon_{\mathrm{m}}}{\varepsilon_{i}+2 \varepsilon_{\mathrm{m}}}}\right)$,

where $f_{i}=V_{i} / V_{\text {total }}$ is the volume fraction of the $i$ th material and $\varepsilon_{\text {eff }}$ is the effective permittivity. The expression for the Bruggeman (1935) rule is

$\sum_{i} f_{i} \frac{\varepsilon_{i}-\varepsilon_{\mathrm{eff}}}{\varepsilon_{i}+2 \varepsilon_{\mathrm{eff}}}=0$.

As an example of a more sophisticated rule, we use the "layered-sphere EMT" introduced by Voshchinnikov \& Mathis (1999). In this case, the effective optical constants $\varepsilon_{\text {eff }}$ are defined as follows (see also Farafonov 2000):

$\varepsilon_{\text {eff }}=\frac{1+2 \alpha / V}{1-\alpha / V}=\frac{\mathcal{A}_{2}}{\mathcal{A}_{1}}$,

where $\alpha$ is the complex electric polarizability and the coefficients $\mathcal{A}_{1}$ and $\mathcal{A}_{2}$ are obtained as a result of multiplication

\footnotetext{
7 The dielectric permittivity is related to the refractive index as $\varepsilon=m^{2}$.
} 
of matrices depending on the optical constants and volume fractions of layers

$$
\begin{aligned}
\left(\begin{array}{l}
\mathcal{A}_{1} \\
\mathcal{A}_{2}
\end{array}\right)= & \left(\begin{array}{cc}
1 & 1 / 3 \\
\varepsilon_{n} & -2 /\left(3 \varepsilon_{n}\right)
\end{array}\right) \\
& \times \prod_{i=n-1}^{2}\left(\begin{array}{cc}
1 / 3\left(\frac{\varepsilon_{i}}{\varepsilon_{i+1}}+2\right) & -2 /\left(9 f_{i}\right)\left(\frac{\varepsilon_{i}}{\varepsilon_{i+1}}-1\right) \\
-f_{i}\left(\frac{\varepsilon_{i}}{\varepsilon_{i+1}}-1\right) & 1 / 3\left(2 \frac{\varepsilon_{i}}{\varepsilon_{i+1}}+1\right)
\end{array}\right) \\
& \times\left(\begin{array}{c}
1 / 3\left(\frac{\varepsilon_{1}}{\varepsilon_{2}}+2\right) \\
-f_{1}\left(\frac{\varepsilon_{1}}{\varepsilon_{2}}-1\right)
\end{array}\right) .
\end{aligned}
$$

The absolute bounds to $\varepsilon_{\text {eff }}$ were given by Wiener $(1910)^{8}$

$\varepsilon_{\mathrm{eff}, \max }=\sum_{i} f_{i} \varepsilon_{i}$

and

$\varepsilon_{\mathrm{eff}, \min }=\left(\sum_{i} \frac{f_{i}}{\varepsilon_{i}}\right)^{-1}$.

For any dielectric particle composition and structure, $\varepsilon_{\mathrm{eff}}$ cannot lie beyond these limits as long as the microstructure dimensions remain small compared with the radiation wavelength. Note that in the composite grain model of Mathis \& Whiffen (1989) the effective refractive index was calculated from Eq. (9), i.e. a maximum of possible refractive indices was taken.

The general condition of EMT applicability is that the size of "inclusions" (in the EMT the particle inhomogeneity is considered in the form of uniformly distributed small inclusions) is small in comparison to the wavelength of incident radiation (Chýlek et al. 2000). The real range of applicability of different rules was shown to be nearly the same (see, e.g., Table 4 in Voshchinnikov 2004).

Now let us discuss how different EMT rules can reproduce the optical properties of layered spheres. Figure 15 (left panel) shows the extinction efficiency factors computed with the exact theory for layered spheres and with the Mie theory using Garnett ${ }^{9}$, Bruggeman, and layered-sphere mixing rules of the EMT (i.e. the effective refractive indices are equal to $m_{\mathrm{eff}}=1.496+0.060 i, m_{\mathrm{eff}}=1.541+0.081 i$ and $m_{\mathrm{eff}}=$ $1.529+0.080 i$, respectively). Wiener's maximum bound is $m_{\text {eff }}=1.604+0.105 i$. Figure 15 (right panel) demonstrates the relative errors of these EMTs. It can be seen that the errors of the Bruggeman and layered-sphere rules are of several percent or better in the considered range of particle sizes. The same is generally true for other efficiency factors and albedo. An exception is the region after the first maximum of the scattering efficiency factor and albedo $\left(x_{\text {porous }} \approx 6-8\right)$ where the relative errors may reach up to $20 \%$. The largest errors occur for the asymmetry parameter, especially for small size parameters. The high accuracy of the layered-sphere rule in the case of very small particles sizes is explained by the fact that it is based on the Rayleigh approximation.

\footnotetext{
${ }^{8}$ These expressions were exactly derived for non-absorbing materials but it seems they can be applied to slightly absorbing materials too.

9 Vacuum was adopted as the matrix material.
}

Other rules of the EMT behave like the Garnett and Bruggeman rules. We note that the general condition of the EMT applicability is not fulfilled for layered particles as "inclusions" (layers) are not small in comparison with the wavelength of incident radiation. However, most rules of the EMT can reproduce the optical properties of layered spheres of any size, if the number of layers is larger than 15-20. This conclusion, however, can be affected by the porosity of particles.

Figure 16 illustrates the applicability of different EMT rules to particles of very high porosity. The cases of other efficiency factors, albedo and asymmetry parameter are similar. The figure demonstrates that the layered-sphere rule rather well reproduces the optical properties of layered spheres as well as the particles with inclusions of different sizes (the errors are smaller than $10-20 \%$ if $x_{\text {porous }} \lesssim 15$ and $\mathcal{P}=0.9$ ). Note that for such particles other rules provide acceptable approximations for intermediate porosity $(\mathcal{P} \lesssim 0.5)$. The Garnett and Bruggeman rules together with the Mie theory rather well approximate the light scattering by particles with inclusions of small sizes. Therefore, all previous models based on the standard EMT-Mie calculations are related to particles composed of subparticles of very small sizes. If the size of subparticles is not small, only the layered-sphere rule can be used for the description of the optical properties of very porous scatterers.

\section{Concluding remarks}

We consider different (including new) computational approaches to calculating the optical properties of composite and porous grains that can be used for the interpretation of observations of interstellar, circumstellar and cometary dust. In our models the particles are represented by multi-layered spheres or pseudospheres with inclusions of one or different sizes. If the number of layers is small, our model of layered spheres coincides with older models of the grains having several coatings. For a large ( $\gtrsim 15-20)$ number of layers, the model of layered spheres can approximate heterogeneous particles consisting of inclusions of different sizes. This gives us a handy way to treat composite grains employing a very simple computational model of multi-layered particles instead of time-consuming DDA calculations.

We make a careful examination of the optical properties of particles of various porosity. Previously, this task was solved using the Mie theory for homogeneous spheres and effective refractive indices derived from different mixing rules of the Effective Medium Theory. It is demonstrated that this approach gives relatively accurate results only if particles have small (Rayleigh) inclusions. Otherwise, the approach becomes unacceptable when the porosity exceeds $\sim 0.5$. An exception is provided by a sophisticated layered-sphere mixing rule, recently suggested by Voshchinnikov \& Mathis (1999), that gives results of acceptable accuracy for particles with non-Rayleigh inclusions as well. Note, however, that our consideration was restricted by spheres, non very absorbing materials and the integral scattering characteristics but not the differential cross sections or elements of the scattering matrix. 
Some astrophysical applications of the model of layered grains (in particular, the possibility to reduce the model dustphase abundances) will be presented in a subsequent paper (Voshchinnikov et al. 2004). Further development of the model of multi-layered particles will involve consideration of nonspherical inhomogeneous grains (see Farafonov et al. 2003 for a review).

Acknowledgements. We are grateful to Bruce Draine and Piotr Flatau for providing DDSCAT 6.0 and to the referee for very careful reading of the paper and recommendations related to its improvement. The work was partly supported by grants of the DFG Research Group "Laboratory Astrophysics" and by grant 1088.2003.2 of the President of the Russian Federation for leading scientific schools. V.I. acknowledges a support by the grant E02-11.0-8 of the Russian Ministry of Education.

\section{References}

Aden, A. L., \& Kerker, M. 1951, J. Appl. Phys., 22, 1242

Andersen, A. C., Sotelo, J. A., Niklasson, G. A., \& Pustovit, V. N. 2003 [arXiv: astro-ph/0310343]

Babenko, V. A., Astafyeva, L. G., \& Kuz'min, V. N. 2003, Electromagnetic scattering in disperse media: inhomogeneous and anisotropic particles (Springer-Praxis)

Bruggeman, D. A. G. 1935, Ann. Phys., 24, 636

Chýlek, P., Videen, G., Geldart, D. J. W., Dobbie, J. S., \& Tso, H. C. W. 2000, in Light Scattering by Nonspherical Particles, ed. M. I. Mishchenko et al. (San Francisco: Academic Press), 274

Dominik, C., Sedlmayr, E., \& Gail, H.-P. 1993, A\&A, 277, 578

Dorschner, J. 1999, in Formation and Evolution of Solids in Space, ed. J. M. Greenberg, \& A. Li (Kluwer), 229

Dorschner, J., \& Henning, Th. 1995, A\&AR, 6, 271

Draine, B. T. 2000, in Light Scattering by Nonspherical Particles, ed. M. I. Mishchenko et al. (San Francisco: Academic Press), 131

Draine, B. T. 2003, ARA\&A, 41, 241

Draine, B. T., \& Flatau, P. J. 2003 [arXiv: astro-ph/0309069]

Draine, B. T., \& Lee, H. M. 1984, ApJ, 285, 89

Ehrenfreund, P. 1999, in Solid Interstellar Matter: the ISO Revolution, ed. L. d'Hendecourt, C. Joblin, \& A. Jones (Berlin: SpringerVerlag), 231

Farafonov, V. G. 2000, Opt. Spectrosc., 88, 441

Farafonov, V. G., Il'in, V. B., \& Prokop'eva, M. S. 2003, JQSRT, 79, 599

Garnett, J. C. M. 1904, Phil. Trans. R. Soc. A, 203, 385

Greenberg, J. M. 1984, Occas. Rep. Roy. Obs. Edinburgh, 12, 1

Greenberg, J. M. 1999, in Formation and Evolution of Solids in Space, ed. J. M. Greenberg, \& A. Li (Kluwer), 53

Greenberg, J. M., \& Hage, J. L. 1991, in Chemistry in Space, ed. J. M. Greenberg, \& V. Pirronello (Kluwer), 363

Gurwich, I., Kleiman, M., Shiloah, N., \& Oaknin, D. 2003, JQSRT, 79,649
Gurwich, I., Shiloah, N., \& Kleiman, M. 2001, JQSRT, 70, 433

Güttler, A. 1952, Ann. Phys., 6, Bd. 11, 65

Hage, J. I., \& Greenberg, J. M. 1990, ApJ, 361, 251

Henning, Th., \& Stognienko, R. 1993, A\&A, 280, 609

Henning, Th., Il'in, V. B., Krivova, N. A., Michel, B., \& Voshchinnikov, N. V. 1999, A\&AS, 136, 405

Jäger, C., Il'in, V. B., Henning, Th., et al. 2003, JQSRT, 79, 765

Johnson, B. R. 1996, Appl. Opt., 35, 3286

Iatì, M. A., Cecchi-Pestellini, C., Williams, D. A., et al. 2001, MNRAS, 322, 749

Kerker, M. 1969, The Scattering of Light and Other Electromagnetic Radiation (New York: Academic Press)

Kolokolova, L., \& Gustafson, B. ̊. S. 2001, JQSRT, 70, 611

Krügel, E., \& Siebenmorgen, R. 1994, A\&A, 288, 929

Laor, A., \& Draine, B. T. 1993, ApJ, 402, 441

Li, A., \& Greenberg, J. M. 1998, A\&A, 331, 291

Lumme, K., \& Rahola, J. 1994, ApJ, 425, 653

Maron, N., \& Maron, O. 2004 [arXiv:astro-ph/0402249]

Mason, C. G., Gehrz, R. D., Jones, T. J., et al. 2001, ApJ, 549, 635

Mathis, J. S. 1996, ApJ, 472, 643

Mathis, J. S., \& Whiffen, G. 1989, ApJ, 341, 808

Mathis, J. S., Rumpl, W., \& Nordsieck, K. H. 1977, ApJ, 217, 425

Ossenkopf, V., \& Henning, Th. 1994, A\&A, 291, 943

Petrov, Yu. A. 1986, Clusters and Small Particles (Moscow: Nauka)

Rouleau, F., \& Martin, P. G. 1991, ApJ, 377, 526

Shifrin, K. S. 1952, Izvestia Academii Nauk SSSR, Ser. Geofizicheskaya, 2, 15

Sihvola, A. H. 1999, Electromagnetic Mixing Formulas and Applications, Institute of Electrical Engineers, Electromagnetic Waves Series 47, London

Snow, T. P., \& Witt, A. N. 1996, ApJ, 468, L65

Spanier, J. E., \& Herman, I. P. 2000, Phys. Rev. B, 61, 10437

Vaidya, D. B., Gupta, R., Dobbie, J. S., \& Chýlek, P. 2001, A\&A, 375, 584

van de Hulst, H. C. 1957, Light scattering by small particles (New York: John Wiley)

Videen, G., \& Chýlek, P. 1998, Optics Comm., 158, 1

Voshchinnikov, N. V. 2004, Astrophys. \& Space Phys. Rev., 12, 1

Voshchinnikov, N. V., \& Mathis, J. S. 1999, ApJ, 526, 257

Voshchinnikov, N. V., Il'in, V. B., Henning, Th., \& Dubkova, D. N. 2004, in preparation

Wickramasinghe, N. C. 1963, MNRAS, 126, 99

Wiener, O. 1910, Berichte über die Verhandlungen der KöniglichSächsischen Gesellschaft der Wisseschaften zu Leipzig, Math.phys. Klasse, 62, 256

Wolff, M. J., Clayton, G. C., \& Gibson, S. J. 1998, ApJ, 503, 815

Wolff, M. J., Clayton, G. C., Martin, P. G., \& Schulte-Ladbeck, R. E. 1994, ApJ, 423, 412

Wu, Z. S., \& Wang, Y. P. 1991, Radio Sci., 26, 1393

Wu, Z. S., Guo, L. X., Ren, K. F., Gouesbet, G., \& Gréhan, G. 1997, Appl. Opt., 36, 5188 